\title{
Automatic pharmacophore model generation using weighted substructure assignments
}

\author{
Andreas Jahn*, H Planatscher, Georg Hinselmann, Nikolas Fechner, A Zell \\ From 5th German Conference on Cheminformatics: 23. CIC-Workshop \\ Goslar, Germany. 8-10 November 2009
}

The generation of a pharmacophore model is a challenging process, which often requires the interaction of medicinal chemists. Given a number of ligands for a specific target, the aim is to identify the pharmacophore patterns that are responsible for the biological activities of chemical compounds. A recent study of optimal assignment methods has shown that the assignment of chemical substructures is able to detect active compounds in a data set [1]. Therefore, we investigated the possibility to use this technique to identify key features of a set of active compounds.

To determine important substructures of active compounds, we integrated $n$ weight factors, where $n$ is the number of substructures. The substructures were defined using the pharmacophore definitions of Phase 3.0 [2]. To define the individual weights of the pharmacophore patterns, we integrated a genetic algorithm which assigns weight factors to the previously defined patterns. The experimental setup was designed as follows: Given a data set with active compounds, the most active compound was selected as query structure for the experiment. The remaining active compounds were inserted into a background data set containing inactive compounds. The genetic algorithm evolved $n$ weights for the pharmacophore patterns of the query structure. To evaluate the fitness of an individual, we performed a single query screening with the weights of the individual. During the optimization process, the BEDROC score [3] is optimized which puts emphasis on the early recognition performance. The result of the genetic algorithm was a weight vector that assigns each pharmacophore feature the weight of the best individual.

We evaluated our approach on a subset of the Directory of Useful Decoys that is suitable for ligand-based virtual screening [1][4]. The query structure was

University of Tübingen, Sand 1, 72076 Tübingen, Germany extracted from the same complexed crystal structure used by Huang et al. [4] to determine the binding site of the protein.

The presented method is able to provide valuable information about key features that are important for the biological activity of a compound. Additionally, information of the protein structure is not needed. Therefore, the method can also be used to derive a pharmacophore model if no protein structure is available (e.g. GPCRs).

Published: 4 May 2010

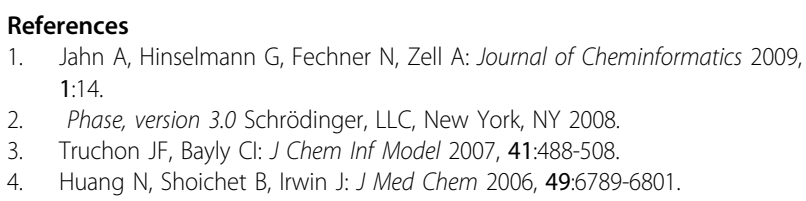

doi:10.1186/1758-2946-2-S1-P42

Cite this article as: Jahn et al:: Automatic pharmacophore model generation using weighted substructure assignments. Journal of Cheminformatics 2010 2(Suppl 1):P42. 